\title{
ALMOST SURE PROPERTIES OF CONTROLLED DIFFUSIONS AND WORST CASE PROPERTIES OF DETERMINISTIC SYSTEMS
}

\author{
Martino Bardi ${ }^{1}$ And Annalisa Cesaroni ${ }^{1}$
}

\begin{abstract}
We compare a general controlled diffusion process with a deterministic system where a second controller drives the disturbance against the first controller. We show that the two models are equivalent with respect to two properties: the viability (or controlled invariance, or weak invariance) of closed smooth sets, and the existence of a smooth control Lyapunov function ensuring the stabilizability of the system at an equilibrium.
\end{abstract}

Mathematics Subject Classification. 93D09, 93E15, 49L25, 49N70.

Received January 11, 2006. Revised October 26, 2006.

Published online October 13, 2007.

\section{INTRODUCTION}

An Ito stochastic differential equation in $\mathbb{R}^{N}$

$$
\mathrm{d} X_{t}=f\left(X_{t}\right) \mathrm{d} t+\sigma\left(X_{t}\right) \mathrm{d} B_{t}, \quad t>0, \quad X_{0}=x,
$$

where $B_{t}$ is an $M$-dimensional Brownian motion and the matrix-valued function $\sigma$ has Lipschitz first derivatives, can be rewritten in the Stratonovich form

$$
\mathrm{d} X_{t}=\left(f\left(X_{t}\right)-\frac{1}{2} \sum_{i=1}^{M} D \sigma^{i}\left(X_{t}\right) \sigma^{i}\left(X_{t}\right)\right) \mathrm{d} t+\sigma\left(X_{t}\right) \circ \mathrm{d} B_{t}, \quad X_{0}=x,
$$

where $\sigma^{i}$ denotes the $i$-th column of $\sigma$ and $D \sigma^{i}$ its Jacobian matrix, and it is well-known that this equation is equivalent in several senses to the deterministic control system

$$
\dot{x}=f(x)-\frac{1}{2} \sum_{i=1}^{M} D \sigma^{i}(x) \sigma^{i}(x)+\sigma(x) \beta(t), \quad x(0)=x,
$$

where $\beta:[0,+\infty) \rightarrow \mathbb{R}^{M}$ varies in some function space. In fact, Stroock and Varadhan $[32,33]$ proved that the support of the measure defined by (1) on the set of functions $[0,1] \rightarrow \mathbb{R}^{N}$ is the closure in uniform topology of

\footnotetext{
Keywords and phrases. Controlled diffusion, robust control, differential game, invariance, viability, stabilization, viscosity solution, optimality principle.

1 Dipartimento di Matematica P. e A., Università di Padova, via Trieste 63, 35131 Padova, Italy; bardi@math.unipd.it; acesar@math . unipd.it
} 
the trajectories of (3) as $\beta$ runs over smooth functions. Doss [19] used this result to prove that a closed set $K$ with smooth boundary is invariant for (1) (i.e., a trajectory of (1) starting in $K$ remains forever in $K$ almost surely) if and only if $K$ is invariant for (3) for all locally integrable $\beta$; very recently Aubin and Doss [3] extended this result to non-smooth sets $K$. Sussmann [34] proved that the solution of the Stratonovich equation (2) coincides with the set of trajectories of (3) as $\beta$ varies among continuous functions. The uniform convergence in the support theorem of Stroock and Varadhan was improved to convergence in Hölder norm in the 90s [1,11,24].

The next step in the comparison between stochastic and deterministic models of dynamical systems with disturbances concerns the controlled diffusion process

$$
\left\{\begin{array}{l}
\mathrm{d} X_{t}=f\left(X_{t}, \alpha_{t}\right) \mathrm{d} t+\sigma\left(X_{t}\right) \mathrm{d} B_{t}, \quad t>0 \\
X_{0}=x
\end{array}\right.
$$

where the control $\alpha$ takes values in a given compact metric space $A$, and the deterministic control system

$$
\left\{\begin{array}{l}
\dot{x}=f(x, \alpha)-\frac{1}{2} \sum_{i=1}^{M} D \sigma^{i}(x) \sigma^{i}(x)+\sigma(x) \beta(t), \quad t>0 \\
x(0)=x
\end{array}\right.
$$

where the disturbance $\beta$ is modelled as a second controller whose goals can be different from those of the first controller $\alpha$. This fits naturally in the theory of differential games and robust control, see [4,10], (the Appendix of [4] by Soravia, in particular), and the references therein. Further motivations come from mathematical finance $[12,25]$.

In the recent paper [18] Da Prato and Frankowska showed that a closed set $K$ is invariant for (CSDE) (i.e., it is almost surely invariant for all stochastic controls $\alpha_{t}$, that is, functions in $A$ progressively measurable with respect to $\left.B_{t}\right)$ if and only if it is invariant for (DS) (i.e., for all $\alpha \in L^{\infty}([0,+\infty), A)$ and $\left.\beta \in L_{l o c}^{1}\left([0,+\infty), \mathbb{R}^{M}\right)\right)$. Their proof is based on estimates on the trajectories of the two systems, as in [19] and [34], under weak regularity assumptions on the data. Note that in this problem the controllers $\alpha$ and $\beta$ cooperate.

The goal of this paper is showing the equivalence of (CSDE) and (DS) with respect to two more properties. Different from the invariance studied in [18], both these properties involve a conflict of the controller against the disturbance, and they are satisfied if the controller can win it. For the deterministic system (DS) this is modelled as a two-person zero-sum differential game.

The first property is the viability, or controlled invariance, or weak invariance, of a closed set $K$ with smooth boundary. For the controlled diffusion (CSDE) this means the existence for all $x \in K$ of a stochastic control $\alpha$ that keeps the system forever in $K$ almost surely, a property that we call a.s. viability. For (DS) it means the existence of a non-anticipating strategy for the first controller that keeps the system in $K$ for all controls $\beta \in L_{l o c}^{1}\left([0,+\infty), \mathbb{R}^{M}\right)$ of the second controller, a property that we call worst case viability (briefly, w.c. viability). Here the controller choosing $\alpha$ is modelled as the first player of the game and the disturbance as the opponent player. A direct approach to the equivalence by estimates on the trajectories seems rather hard in this case, and we make instead a comparison between geometric characterizations of the properties, usually called viability theorems. Such a theorem for deterministic differential games with bounded controls and disturbances was found by Cardaliaguet [14] and it involves only the vector fields of the system (DS) and the normal to the boundary (see also [8] for a different proof). We provide in Section 2 an extension of this result to systems affected by unbounded disturbances, with a new proof relying on viscosity solutions of H-J equations $[4,17,20]$ and optimality principles $[29,31]$. The geometric characterization of a.s. viability of (CSDE) due to the first author and Jensen [9] uses also the second fundamental form of the boundary (see also [2,13] and the references therein for earlier results). In Section 2 we prove that these two conditions are equivalent, thus a.s. viability of $K$ for (CSDE) is equivalent to w.c. viability for (DS).

The second property is the stabilizability of the system to an equilibrium position, and we do it for both notions of plain (Lyapunov) stability and of asymptotic stability. Again, for (CSDE) stabilizability means the existence of a stochastic control $\alpha$ that stabilizes the system almost surely, for (DS) it means the existence 
of a strategy that does the job for all disturbances $\beta \in L_{l o c}^{1}\left([0,+\infty), \mathbb{R}^{M}\right)$ and we call it worst case (briefly, w.c.) stability. The almost sure stabilizability of (CSDE) was introduced by the authors in [5] and studied by means of suitable Lyapunov functions satisfying 2 nd order partial differential inequalities in the viscosity sense, see also [16] for converse Lyapunov theorems. We remark that this property is stronger than stability in probability and pathwise stability, and in fact it is never verified by a nondegenerate process. Worst case stability for bounded disturbances was studied by Soravia $[28,29]$ by means of Lyapunov functions satisfying first order partial differential inequalities in the viscosity sense, and he also treated the case of unbounded noise in the framework of $H^{\infty}$ control [30,31]. In Section 3 we extend his results on worst case stability to unbounded disturbances and show that the existence of a w.c. Lyapunov function is equivalent to w.c. stability. Then we prove that a $C^{2}$ function is a Lyapunov function for the a.s stabilizability of (CSDE) if and only if it is a Lyapunov function for the w.c. stability of (DS).

Let us recall some of the many issues related to these properties. Invariance and reachability properties of (CSDE) arise in financial mathematics, see $[3,26]$ and the references therein, and in the geometric partial differential equations describing the motion of fronts, see Soner and Touzi [27]. They are also used, together with the viability and reachability properties of (DS), in the study of strong maximum principles for nonlinear degenerate elliptic equations [6,7], in a similar way as the support theorem was used by Stroock and Varadhan for linear PDEs. The notion of w.c. stabilizability is related to the robust stabilization and robust control Lyapunov function studied by Freeman and Kokotovic [21] for bounded disturbances, see also [23] and the references therein.

We end this introduction with a list of assumptions that will hold throughout the paper and with some basic definitions and notations. Given a compact metric space $A$, the data $f: \mathbb{R}^{N} \times A \rightarrow \mathbb{R}^{N}$ and $\sigma$, which maps $\mathbb{R}^{N}$ to the space of $N \times M$ matrices, are continuous functions which satisfy

$$
\begin{gathered}
|f(x, \alpha)-f(y, \alpha)| \leq K|x-y|, \quad \forall x, y \in \mathbb{R}^{N}, \quad \forall \alpha \in A \\
\sigma \in C^{1,1}, \quad\left\|D \sigma^{i}(x)-D \sigma^{i}(y)\right\| \leq K|x-y| \quad i=1, \ldots, M \quad \forall x, y \in \mathbb{R}^{N} .
\end{gathered}
$$

The class of admissible controls for (CSDE) is the class $\mathcal{U}$ of strict controls with values in $A$, as defined in [22], Definition 2.2. The admissible controls and disturbances for (DS) are given by the two sets $\mathcal{A}:=L^{\infty}([0,+\infty), A)$ and $\mathcal{B}:=L_{\text {loc }}^{1}\left([0,+\infty), \mathbb{R}^{M}\right)$. The admissible strategies $\Gamma$ for the controller (i.e., the first player) are functionals $\gamma: \mathcal{B} \rightarrow \mathcal{A}$ with the property of being non-anticipating:

$$
\forall t>0, \quad \beta=\bar{\beta} \text { a.e. in }[0, t] \text { implies } \gamma(\beta)=\gamma(\bar{\beta}) \text { a.e. in }[0, t] .
$$

We define $a(x):=\frac{1}{2} \sigma(x) \sigma(x)^{T}$, where ${ }^{T}$ denotes the transpose. Finally, we assume

$$
\{f(x, \alpha): \alpha \in A\} \quad \text { is convex for all } x \in \mathbb{R}^{N} .
$$

This is a classical condition to ensure existence of optimal controls in problems satisfying standard regularity assumptions, and if it is not satisfied one can get it by considering relaxed controls, see [22].

\section{A.s. Stochastic Viability is EQUivalent to W.C. Deterministic Viability}

We begin recalling the definition of stochastic and worst case viability of a set. Almost sure viability was first studied by Aubin and Da Prato, see $[2,13]$ and the references therein. Here we use a geometric characterization found by the first author and Jensen [9].

Definition 2.1 (a.s. viability). A closed set $K \subset \mathbb{R}^{N}$ is a.s. viable or controlled invariant or weakly invariant for the stochastic system $(C S D E)$ if for all initial points $x \in K$ there exists an admissible control $\alpha \in \mathcal{U}$ such that the corresponding trajectory $X$. satisfies $X_{t} \in K$ for all $t>0$ almost surely. 
Definition 2.2 (w.c. viability). A closed set $K \subset \mathbb{R}^{N}$ is worst case viable with respect to the system (DS) if for every $x \in K$ there exists a strategy $\gamma \in \Gamma$ such that the corresponding trajectory $x(\cdot)=x(\cdot ; \gamma(\beta), \beta)$ satisfies $x(t) \in K$ for every $t \geq 0$ and every admissible control $\beta \in \mathcal{B}$.

The characterization of a.s. viability in [9] is in terms of a generalized second order normal cone introduced in [8]. We recall the definition.

Definition 2.3 (second order normal cone). Let $K \subseteq \mathbb{R}^{N}$ a closed set. The second order normal cone at $x \in K$ is

$$
\begin{aligned}
\mathcal{N}_{K}^{2}(x):= & \left\{(p, Y) \in \mathbb{R}^{N} \times S(N): \text { for } y \rightarrow x, y \in K,\right. \\
& \left.p \cdot(y-x)+\frac{1}{2}(y-x) \cdot Y(y-x) \geq o\left(|y-x|^{2}\right)\right\}
\end{aligned}
$$

where $S(N)$ is the set of symmetric $N \times N$ matrices.

Note that, if $(p, Y) \in \mathcal{N}_{K}^{2}(x)$ and $x \in \partial K$, the vector $p$ is a Bony (i.e. proximal) interior normal to the set $K$ at $x$. In particular, if $\partial K$ is a smooth surface in a neighborhood of $x, p /|p|$ is the interior unit normal and $Y$ is related to the second fundamental form of $\partial K$ at $x$, see [8].

Here is the Nagumo-type geometric characterization of viability, see [9], Theorem 1 and Proposition 1.

Theorem 2.1. Let $K \subset \mathbb{R}^{N}$ be a closed set. Then $K$ is a.s. viable for $(C S D E)$ if and only if

$$
\forall x \in \partial K, \forall(p, Y) \in \mathcal{N}_{K}^{2}(x), \exists \alpha \in A: \quad f(x, \alpha) \cdot p+\operatorname{trace}(a(x) Y) \geq 0 .
$$

Moreover,

$$
\sigma^{i}(x) \cdot p=0 \quad \forall i=1, \ldots, M .
$$

Now we turn to the geometric characterization of w.c. viability. The first result of this kind was given by Cardaliaguet [14] in terms of the Severi-Bouligand tangent cone and of its dual cone. Later the first author and Goatin [8] gave a characterization of w.c. viability in terms of the interior Bony (i.e. proximal) normal cone, namely,

$$
\mathcal{N}_{K}^{1}(x):=\left\{p \in \mathbb{R}^{N}: \text { for } y \rightarrow x, y \in K, p \cdot(y-x) \geq o(|y-x|)\right\} .
$$

These characterizations were proved in the case in which both the controls and the disturbances acting in the differential game take value in a compact metric space. We provide next the extension of this result to our system (DS), where the set of disturbances is $\mathbb{R}^{M}$. Our proof is different from those in $[8,14]$, and it relies on the superoptimality principle in the Appendix.

Theorem 2.2. Let $K \subset \mathbb{R}^{N}$ be a closed set. Then $K$ is viable for $(D S)$ if and only if

$$
\begin{gathered}
\forall x \in \partial K, \forall p \in \mathcal{N}_{K}^{1}(x), \forall \beta \in \mathbb{R}^{M}, \exists \alpha \in A: \\
\left(f(x, \alpha)-\frac{1}{2} \sum_{i=1}^{M} D \sigma^{i}(x) \sigma^{i}(x)+\sigma(x) \beta\right) \cdot p \geq 0 .
\end{gathered}
$$

Proof. We define the LSC function

$$
v_{K}(x):= \begin{cases}1 & x \notin K \\ 0 & x \in K\end{cases}
$$

It is easy to check that, for $x \in \partial K$, the subdifferential $D^{-} v_{K}(x)=-\mathcal{N}_{K}^{1}(x)$, whereas $D^{-} v_{K}(x)=\{0\}$ for $x \notin \partial K$ (we refer to $[4,17]$ for the definitions of subdifferential and viscosity supersolution). So the differential condition (9) holds if and only if $v_{K}$ is a viscosity supersolution to

$$
\inf _{\beta \in \mathbb{R}^{M}} \max _{\alpha \in A}\left(-f(x, \alpha)+\frac{1}{2} \sum_{i=1}^{M} D \sigma^{i}(x) \sigma^{i}(x)-\sigma(x) \beta\right) \cdot D v_{K}(x)=0 \quad \text { in } \mathbb{R}^{N} .
$$


Moreover it is easy to check that $K$ is viable if and only if $v_{K}$ satisfies the following superoptimality principle:

$$
v_{K}(x)=\inf _{\gamma \in \Gamma} \sup _{\beta \in \mathcal{B}} \sup _{t \geq 0} v_{K}(x(t ; \gamma(\beta), \beta)) .
$$

Therefore we obtain the conclusion if we show that a LSC function $W$ is a viscosity supersolution to (10) if and only if it satisfies (11). The direct part is proved in Proposition A.1 of the Appendix, whereas the converse part relies on standard methods in viscosity solution theory (see, e.g., [4], Chap. VIII).

We prove now the equivalence between a.s. viability and w.c. viability.

Proposition 2.1. Consider $K=\bar{\Omega}$, where $\Omega \subseteq \mathbb{R}^{N}$ is an open set whose boundary $\partial \Omega$ is a $C^{2}$ hypersurface. Then $K$ is a.s. viable with respect to the stochastic system (CSDE) if and only if it is w.c. viable with respect to the deterministic system (DS).

Proof. We denote with $\vec{n}(x)$ the interior normal at $x \in \partial \Omega$. Under the regularity assumptions on $K$, we can express the second order normal cone in terms of the gradient and the Hessian matrix of any function $\rho$ of class $C^{2}$ in a neighborhood of $\partial K$ and such that $\rho(x)=0$ and $D \rho(x)=\vec{n}(x)$ for $x \in \partial K$ (e.g., the signed distance function from $\partial K)$. Indeed, for $x \in \partial K, \mathcal{N}_{K}^{2}(x)=\left\{(D \rho(x), Y) \mid Y \geq D^{2} \rho(x)\right\}$.

Assume that $K$ is a.s. viable for (CSDE). Then, by Theorem 2.1,

$$
\forall x \in \partial K \exists \alpha \in A: f(x, \alpha) \cdot D \rho(x)+\operatorname{trace}\left(a(x) D^{2} \rho(x)\right) \geq 0
$$

and moreover, by $(8), \sigma^{i}(x) \cdot D \rho(x)=0$ for every $i=1, \ldots, M$. Now we observe that

$$
0=\sigma^{i}(x) D\left[\sigma^{i}(x) \cdot D \rho(x)\right]=\sigma^{i}(x)^{T} D^{2} \rho(x) \sigma^{i}(x)+D \sigma^{i}(x) \sigma^{i}(x) \cdot D \rho(x) .
$$

We sum over $i$ and compute

$$
\frac{1}{2} \sum_{i=1}^{M} \sigma^{i}(x)^{T} D^{2} \rho(x) \sigma^{i}(x)=\frac{1}{2} \operatorname{trace}\left(\sigma(x)^{T} D^{2} \rho(x) \sigma(x)\right)=\operatorname{trace}\left(a(x) D^{2} \rho(x)\right) .
$$

By substituting into (12) we get

$$
\forall x \in \partial K \exists \alpha \in A: \sigma^{i}(x) \cdot D \rho(x)=0 \quad f(x, \alpha) \cdot D \rho(x)-\frac{1}{2} \sum_{i=1}^{M} D \sigma^{i}(x) \sigma^{i}(x) \cdot D \rho(x) \geq 0,
$$

from which we deduce that for every $x \in \partial K$ there exists $\alpha \in A$ such that, for every $\beta \in \mathbb{R}^{M}$,

$$
\left(f(x, \alpha)-\frac{1}{2} \sum_{i=1}^{M} D \sigma^{i}(x) \sigma^{i}(x)+\sigma(x) \beta\right) \cdot D \rho(x) \geq 0 .
$$

By Theorem 2.2 the last condition is equivalent to the viability of $K$ for (DS).

Assume now that $K$ is viable for (DS). Then condition (14) holds for some $\alpha \in A$ and every $\beta \in \mathbb{R}^{M}$. From this we deduce immediately that $\sigma^{i}(x) \cdot D \rho(x)=0$. Now we substitute (13) in (14) and we get (12), which is equivalent to the viability of $K$ for (CSDE).

Example 2.1. Consider the 1-dimensional stochastic control system

$$
\mathrm{d} X_{t}=X_{t}\left(1-\alpha_{t}-\frac{X_{t}}{k}\right) \mathrm{d} t+\sigma\left(X_{t}\right) \mathrm{d} B_{t}
$$


modelling the density of a specie in an ecosystem, where the control $\alpha$ represent the harvesting factor and takes values in $[0,1]$. Note that when there is neither disturbance nor exploitation, i.e., $\sigma \equiv 0$ and $\alpha \equiv 0$, the dynamics reduces to a standard logistic equation with carrying capacity $k>0$. The corresponding deterministic model is

$$
\dot{x}=x\left(1-\alpha(t)-\frac{x}{k}\right)-\frac{1}{2} \sigma^{\prime}(x) \sigma(x)+\sigma(x) \beta(t)
$$

with $\alpha(t) \in[0,1]$ and $\beta(t) \in \mathbb{R}$.

The viability of an interval $[b, c], 0 \leq b \leq c$, means the existence of an exploitation policy that keeps the population between the levels $b$ and $c$ almost surely (resp., for all disturbances $\beta$ ). By Proposition 2.1 this property can be checked on either one of the two models. Let us do it for the deterministic model, by means of Theorem 2.2. The interval $[b, c]$ is w.c. viable if and only if for all $\beta \in \mathbb{R}$ there is $\alpha \in[0,1]$ such that the function

$$
\phi(x):=x\left(1-\alpha-\frac{x}{k}\right)-\frac{1}{2} \sigma^{\prime}(x) \sigma(x)+\sigma(x) \beta
$$

is nonnegative at $x=b$, and for all $\beta \in \mathbb{R}$ there is $\alpha \in[0,1]$ such that $\phi(c) \leq 0$. And this occurs if and only if

$$
\sigma(b)=\sigma(c)=0 \quad \text { and } \quad b \leq k
$$

In this very simple example the proof of the a.s. viability could also be easily got directly by means of Theorem 2.1. However, in higher dimensions (e.g., in predator-prey models or in competing species models), the calculation of the second order normal cone might be hard and one could prefer the criterion for deterministic systems of Theorem 2.2 that involves only the unit normals at the boundary.

Note that in this example no interval $[b, c]$ with $b>0$ is invariant for all control functions, even when $\sigma \equiv 0$, because $\phi(b)<0$ for $\alpha$ sufficiently close to 1 . Therefore the invariance theory of [18] does not give any sufficient condition of viability in this case.

\section{A.s. STABILIZABILITY IS EQUIVALENT TO W.C. STABILITY}

We recall the notion of almost sure (open loop) stabilizability for stochastic systems and the notion of stability in the worst case for deterministic systems.

Definition 3.1 (comparison functions). $\mathcal{K}$ denotes the class of continuous real functions $\kappa$ strictly increasing and such that $\kappa(0)=0$. $\mathcal{K} \mathcal{L}$ denotes the class of continuous functions $\lambda:[0,+\infty) \times[0,+\infty) \rightarrow[0,+\infty)$ which are strictly increasing in the first variable, strictly decreasing in the second variable, and satisfy

$$
\lambda(0, t)=0 \quad \forall t \geq 0 \quad \lim _{t \rightarrow+\infty} \lambda(r, t)=0 \quad \forall r \geq 0
$$

Definition 3.2 (a.s. stabilizability). The system (CSDE) is almost surely (open-loop Lyapunov) stabilizable at the origin if there exists $\kappa \in \mathcal{K}$ and $\delta_{o}>0$ such that for any starting point $x$ with $|x| \leq \delta_{o}$

$$
\exists \alpha \in \mathcal{U}: \quad\left|X_{t}\right| \leq \kappa(|x|) \quad \forall t \geq 0 \text { a.s. }
$$

If, in addition, $\lim _{t \rightarrow+\infty} X_{t}=0$ a.s., the system is a.s. (open loop) asymptotically stabilizable, or a.s. asymptotically controllable, locally if this occurs for every $x$ in a neighborhood of 0 , globally if it does for every $x$.

Remark. The a.s. stabilizability implies that the origin is a controlled equilibrium, i.e., $\exists \bar{\alpha} \in A: f(0, \bar{\alpha})=$ $0, \sigma(0)=0$. For more details, see [5].

Definition 3.3 (w.c. stability). The system (DS) is worst case stable (w.c. stable) at the origin if there is $\kappa \in \mathcal{K}$ and $\delta_{0}>0$ such that for any starting point $x$ with $|x| \leq \delta_{0}$

$$
\exists \gamma \in \Gamma: \quad|x(t ; \gamma(\beta), \beta)| \leq \kappa(|x|) \quad \text { for every } \beta \in \mathcal{B}, \forall t \geq 0 \text {. }
$$


If moreover there exists $\lambda \in \mathcal{K} \mathcal{L}$, independent of $x$ and $\gamma$, such that

$$
|x(t ; \gamma(\beta), \beta)| \leq \lambda(|x|, t), \quad \text { for every } \beta \in \mathcal{B}, \forall t \geq 0,
$$

then the system is worst case asymptotically stable (w.c. asymptotically stable).

In [5] and [16] we characterized the a.s. stabilizability property by means of appropriate control Lyapunov functions.

Definition 3.4 (a.s. control Lyapunov function). Let $\mathcal{O} \subseteq \mathbb{R}^{N}$ be an open set containing the origin. A function $V: \mathcal{O} \rightarrow[0,+\infty)$ is an a.s. control Lyapunov function for the system $(C S D E)$ if

(i) $V$ is LSC and continuous at 0 ;

(ii) $V$ is positive definite, i.e., $V(0)=0$ and $V(x)>0$ for all $x \neq 0$;

(iii) $V$ is proper, i.e., the sublevel sets $\{x \mid V(x) \leq \mu\}$ are bounded $\forall \mu \in[0,+\infty)$;

(iv) $V$ is a viscosity supersolution in $\mathcal{O} \backslash\{0\}$ of the following system of PDEs and inequalities

$$
\left\{\begin{array}{l}
\max _{\alpha \in A}\{-D V(x) \cdot f(x, \alpha)\}-\operatorname{trace}\left[a(x) D^{2} V(x)\right] \geq l(x) \\
\sigma^{i}(x) \cdot D V(x)=0 \quad \forall i
\end{array}\right.
$$

where $l: \mathcal{O} \rightarrow \mathbb{R}$ is a Lipschitz continuous nonnegative function such that $l(0)=0$. When $l$ is positive definite, we say that $V$ is a strict a.s. control Lyapunov function for $(C S D E)$.

The system of PDEs and inequalities (17) is not a standard Hamilton-Jacobi-Bellman inequality arising in stochastic optimal control. In fact, the system of equations $\sigma(x) \cdot D V(x)=0$ implies that we are allowing only functions $V$ whose level sets are tangential to the diffusion matrix in a generalized sense.

The characterization of almost sure stabilizability in terms of Lyapunov functions found in $[5,16]$ is summarized in the next result.

Theorem 3.1. Assume (4), (6). Then

(i) the system $(C S D E)$ is almost surely stabilizable in a neighborhood of the origin if and only if there exists an a.s. Lyapunov function $V$ for the system;

(ii) the system $(C S D E)$ is almost surely asymptotically stabilizable in a neighborhood of the origin if and only if there exists a strict a.s. Lyapunov function $V$ for the system.

Remark. The key observations for such a Lyapunov characterization is that, as in the deterministic case, the a.s. stabilizability is equivalent to the existence of a basis of viable neighborhoods of the equilibrium and that the sublevel sets of every a.s. control Lyapunov function form a basis of viable neighborhoods of the equilibrium. The proof of both direct and converse theorems is based on the analysis of viability properties of sublevel sets of viscosity supersolutions to (17), using the characterization of viability recalled in the previous section. The direct Lyapunov theorems are proved in [5] and the converse results in [16]. We recall only the explicit construction of the Lyapunov functions. Define

$$
V(x):=\inf \left\{r \mid \exists \bar{\alpha} \text { admissible control such that }\left|\bar{X}_{t}\right| \leq r \text { almost surely } \forall t \geq 0\right\}
$$

or, equivalently,

$$
V(x):=\inf _{\alpha \in \mathcal{U}} \sup _{t \geq 0} \operatorname{ess} \sup _{\omega \in \Omega}\left|X_{t}^{\alpha}\right| .
$$

This is an a.s. Lyapunov function for an a.s. stabilizable system. For an a.s. asymptotically stabilizable system in a bounded set $\mathcal{O}$, in [16] we construct a Lipschitz continuous positive definite function $l$ such that the function defined as

is a strict a.s. Lyapunov function.

$$
V(x):=\inf _{\alpha \in \mathcal{U}} \operatorname{ess} \sup _{\omega \in \Omega} \int_{0}^{+\infty} l\left(X_{t}^{\alpha}\right) \mathrm{d} t
$$


We present next a complete Lyapunov-like characterization of worst case stability by means of control Lyapunov functions, see $[21,23]$ and the references therein.

Definition 3.5 (w.c. control Lyapunov function). A function $W: \mathbb{R}^{N} \rightarrow[0,+\infty)$ is a worst case control Lyapunov function for the system (DS) if

(i) $W$ is LSC and continuous at 0 ,

(ii) $W$ is positive definite,

(iii) $W$ is proper,

(iv) $W$ is a viscosity supersolution of

$$
\inf _{\beta \in \mathbb{R}^{M}} \max _{\alpha \in A}\left(-f(x, \alpha)+\frac{1}{2} \sum_{i=1}^{M} D \sigma^{i}(x) \sigma^{i}(x)-\sigma(x) \beta\right) \cdot D W(x) \geq l(x),
$$

where $l: \mathbb{R}^{N} \rightarrow \mathbb{R}$ is a nonnegative Lipschitz continuous function such that $l(0)=0$. If, moreover, $l$ is positive definite, we say that $W$ is a strict w.c. control Lyapunov function.

The following characterization of w.c. stability in terms of w.c. Lyapunov functions extends some results by Soravia $[28,29]$ to the case of unbounded disturbances. In particular it gives a formula for the construction of a Lyapunov function in the case of asymptotic w.c. stability.

Theorem 3.2. Assume (4), (6), then the system (DS) is w.c. stable (respectively, w.c. asymptotically stable) at the origin if and only if there exists a w.c. control Lyapunov function $W$ (respectively, a strict w.c. control Lyapunov function).

Proof. The proofs of the direct part rely on the following superoptimality principle for viscosity supersolution $W$ of the Hamilton-Jacobi-Bellman-Isaacs equation (18), see Proposition A.1 in the Appendix:

$$
W(x)=\inf _{\gamma \in \Gamma} \sup _{\beta \in \mathcal{B}} \sup _{t \geq 0}\left[W(x(t ; \gamma(\beta), \beta))+\int_{0}^{t} l(x(s ; \gamma(\beta), \beta)) \mathrm{d} s\right] .
$$

When $l$ is merely non-negative we deduce from this, using the properties of $W$, that for every $\varepsilon>0$ there exists $\delta$ such that for $|x| \leq \delta$ there exists an admissible strategy $\gamma$ such that for every admissible disturbance $\beta$ the corresponding trajectory satisfies $|x(t ; \gamma(\beta), \beta)| \leq \varepsilon$ for every $t$. This gives the w.c. stability. When $l$ is positive definite, using (19) again, the properties of $W$ and standard properties of the trajectories of the system, we get in addition that $x(t ; \gamma(\beta), \beta) \rightarrow 0$ as $t \rightarrow+\infty$, and this gives the w.c. asymptotic stability. For more details see [29] for the w.c. stability and [28] for the w.c. asymptotic stability.

Conversely, given a w.c. stable differential system, a candidate Lyapunov function $W$ is constructed as follows: it is the lower semicontinuous envelope $W_{*}$ of the lower value function

$$
W(x):=\inf _{\gamma \in \Gamma} \sup _{\beta \in \mathcal{B}} \sup _{t \geq 0}|x(t ; \gamma(\beta), \beta)| .
$$

It is immediate to check the first three conditions in Definition 3.5, the proof of the differential condition relies on standard methods in viscosity solution theory (see, e.g., [4], Chap. VIII).

We assume now that the system is w.c. asymptotically stable. Then it is possible to construct an appropriate running cost $l$ which is positive definite and Lipschitz continuous by using the decay estimate (16). For more details we refer to [15], Section 1.6. Moreover, by the definition of $l$ we get that the value function $W$ of the infinite horizon problem

$$
W(x):=\inf _{\gamma \in \Gamma} \sup _{\beta \in \mathcal{B}} \int_{0}^{+\infty} l(|x(t ; \gamma(\beta), \beta)|) \mathrm{d} t
$$

is finite. It is quite standard to prove, using the properties of $l$, that the LSC envelope of $W$ is a w.c. strict Lyapunov function for the system (for the details see [4], Chap. VIII and [15], Sect. 1.6). 
Example 3.1. Let us look for conditions under which the system (DS) has a radial w.c. control Lyapunov function, i.e., $W(x)=w(|x|)$ for some $C^{1}$ function $w:[0,+\infty) \rightarrow[0,+\infty)$ with $w^{\prime}(r)>0$ for $r>0$. We assume for simplicity that the disturbance is scalar, so $M=1$ and $\sigma(x) \in \mathbb{R}^{N}$. Then the left hand side of (18) is

$$
\inf _{\beta \in \mathbb{R}} \max _{\alpha \in A}\left(-f(x, \alpha)+\frac{1}{2} D \sigma(x) \sigma(x)-\sigma(x) \beta\right) \cdot x \frac{w^{\prime}(|x|)}{|x|},
$$

which is $-\infty$ if $\sigma(x) \cdot x \neq 0$. Therefore we assume $\sigma(x) \cdot x=0$, and differentiate it to get

$$
\sum_{k=1}^{N} \frac{\partial \sigma_{k}(x)}{\partial x_{j}} x_{k}=-\sigma_{j}(x) \quad \text { for } j=1, \ldots, N .
$$

We multiply by $\sigma_{j}$ and add over $j$ to obtain

$$
D \sigma(x) \sigma(x) \cdot x=-|\sigma(x)|^{2} .
$$

In conclusion, (DS) with $M=1$ admits a radial $C^{1}$ w.c. Lyapunov function if and only if

$$
\sigma(x) \cdot x=0 \quad \text { and } \quad \max _{\alpha \in A}(-f(x, \alpha) \cdot x) \geq \frac{|\sigma(x)|^{2}}{2} .
$$

This condition is independent of the choice of $w$. Moreover, if

$$
l(x):=\left[\max _{\alpha \in A}(-f(x, \alpha) \cdot x)-\frac{|\sigma(x)|^{2}}{2}\right] \frac{w^{\prime}(|x|)}{|x|}
$$

is Lipschitz in a neighborhood of 0 , positive for $x \neq 0$ and $l(x) \rightarrow 0$ as $x \rightarrow 0$, then $W$ is a strict Lyapunov function.

Example 3.2. We consider the case that the system $\dot{x}=f(x, \alpha)$, i.e., (DS) with $\sigma \equiv 0$, is asymptotically stabilizable. By the converse Lyapunov theorem of Sontag (see, e.g., [15]), there exists a strict continuous control Lyapunov function for this subsystem, i.e., there exists a proper function $V$ satisfying in viscosity sense in $\mathbb{R}^{N} \backslash\{0\}$, for some positive definite continuous function $L$,

$$
\max _{\alpha \in A}(-f(x, \alpha) \cdot D V(x))-L(x) \geq 0 .
$$

Under this condition, the left hand side of (18) is

$$
\inf _{\beta \in \mathbb{R}^{M}} \max _{\alpha \in A}\left(-f(x, \alpha)+\frac{1}{2} D \sigma(x) \sigma(x)-\sigma(x) \beta\right) \cdot D V(x)= \begin{cases}-\infty, & \sigma \cdot D V \neq 0, \\ \geq L+\frac{1}{2} D \sigma \sigma \cdot D V, & \sigma \cdot D V=0 .\end{cases}
$$

So $V$ is also a w.c. control Lyapunov function for (DS) if and only if it satisfies in viscosity sense

$$
\sigma(x) \cdot D V(x)=0 \quad \text { and } \quad L(x)+\frac{1}{2} D \sigma(x) \sigma(x) \cdot D V(x) \geq 0 .
$$

The main result of this section is the equivalence between smooth a.s. Lyapunov functions for the system (CSDE) and smooth w.c. Lyapunov functions for the system (DS).

Proposition 3.1. Let $V: \mathbb{R}^{N} \rightarrow \mathbb{R}$ a $C^{2}$ function. Then $V$ is an a.s. control Lyapunov function (respectively, strict a.s. control Lyapunov function) for the stochastic system (CSDE) if and only if it is a w.c. control Lyapunov function (respectively, strict w.c. control Lyapunov function) for the deterministic system (DS). 
Proof. We assume there exists a $C^{2}$ a.s. Lyapunov function $V$ for the system (CSDE) according to Definition 3.4. Then for every $x$ there exists $\bar{\alpha} \in A$ such that

$$
\sigma^{i}(x) \cdot D V(x)=0 \quad \text { and } \quad-D V(x) \cdot f(x, \bar{\alpha})-\frac{1}{2} \operatorname{trace}\left(\sigma(x)^{T} D^{2} V(x) \sigma(x)\right) \geq l(x)
$$

where $l \geq 0$. From the equality in (22) we get

$$
0=\sigma^{i}(x) D\left[\sigma^{i}(x) \cdot D V(x)\right]=\sigma^{i}(x)^{T} D^{2} V(x) \sigma^{i}(x)+D \sigma^{i}(x) \sigma^{i}(x) \cdot D V(x),
$$

and we plug it into the inequality in (22) to deduce that for every $x$ there exists $\bar{\alpha} \in A$ such that for every $\beta \in \mathbb{R}^{M}$

$$
\left(-f(x, \bar{\alpha})+\frac{1}{2} \sum_{i=1}^{M} D \sigma^{i}(x) \sigma^{i}(x)-\sigma(x) \beta\right) \cdot D V(x) \geq l(x) .
$$

This implies that $V$ is a supersolution of

$$
\max _{\alpha \in A} \inf _{\beta \in \mathbb{R}^{M}}\left(-f(x, \alpha)+\frac{1}{2} \sum_{i=1}^{M} D \sigma^{i}(x) \sigma^{i}(x)-\sigma(x) \beta\right) \cdot D V(x) \geq l(x)
$$

and therefore it is a w.c. Lyapunov function.

Assume now that $V$ is a $C^{2}$ w.c. Lyapunov function, i.e., a supersolution of (25). Then for every $x$ there exists $\bar{\alpha} \in A$ such that for all $\beta \in \mathbb{R}^{M}(24)$ is verified. This implies that $D V(x) \cdot \sigma^{i}(x)=0$ for all $i=1, \ldots, M$. Now (23) gives that for every $x$ there exists $\bar{\alpha} \in A$ such that (22) holds. Therefore $V$ is an a.s. Lyapunov function for the system (CSDE).

Remark. We do not know the minimal regularity of $V$ under which the last proposition remains true. If it held for merely semicontinuous $V$ we would get the equivalence of a.s. stabilizability and w.c. stability.

Example 3.3. Let $W(x)=w(|x|)$ be a radial function, with $w:[0,+\infty) \rightarrow[0,+\infty)$ of class $C^{2}$ and such that $w^{\prime}(r)>0$ for $r>0$. In Example 3.1 we showed that $W$ is a w.c. control Lyapunov function for the system (DS) if and only if condition (20) is satisfied. Under the same condition $W$ is also an a.s. control Lyapunov function for the system (SDE): this follows from Proposition 3.1, and it can also be checked by direct computation (see Example 4 in [5]).

Example 3.4. We consider the case that (CSDE) is a stochastic perturbation of an asymptotically stabilizable system $\dot{x}=f(x, \alpha)$. We assume that the unperturbed system admits a $C^{2}$ control Lyapunov function $V$ and we wonder under which conditions $V$ remains an a.s. control Lyapunov function for (CSDE). By Proposition 3.1 a necessary and sufficient condition is (21) derived in Example 3.2; note that it involves only the gradient of $V$. An equivalent condition involving the second derivatives of $V$ is the following:

$$
\sigma(x) \cdot D V(x)=0 \quad \text { and } \quad L(x)-\frac{1}{2} \operatorname{trace}\left(\sigma(x) \sigma^{T}(x)\right) D^{2} V(x) \geq 0,
$$

that was found in Example 2 of [5].

\section{A. Appendix. A superoptimality principle}

This appendix is devoted to the superoptimality principle for viscosity supersolution of Hamilton-JacobiIsaacs equations with possibly infinite Hamiltonian. The proof is an adaptation of a result obtained by Soravia (Lem. 2.2 in [31]) in a different setting, namely, for a problem of $\mathcal{H}^{\infty}$ control. Therefore we give here an outline of the proof and we refer to [31] for some technical details. We recall that we are still assuming the conditions listed in the Introduction. 
Proposition A.1. Let $W: \mathbb{R}^{N} \rightarrow \mathbb{R}$ be a lower semicontinuous supersolution of

$$
\inf _{\beta \in \mathbb{R}^{M}} \max _{\alpha \in A}\left(-f(x, \alpha)+\frac{1}{2} \sum_{i=1}^{M} D \sigma^{i}(x) \sigma^{i}(x)-\sigma(x) \beta\right) \cdot D W(x)-l(x)=0
$$

where $l: \mathbb{R}^{N} \rightarrow \mathbb{R}$ is a nonnegative, Lipschitz continuous function. Then $W$ satisfies the following superoptimality principle:

$$
W(x)=\inf _{\gamma \in \Gamma} \sup _{\beta \in \mathcal{B}} \sup _{t \geq 0}\left[W(x(t ; \gamma(\beta), \beta))+\int_{0}^{t} l(x(s ; \gamma(\beta), \beta)) \mathrm{d} s\right] .
$$

Proof. We may assume without loss of generality that $l \equiv 0$. Indeed, if it is not so, we add a new variable to the dynamics

$$
\begin{array}{ll}
\dot{x}=f(x)-\frac{1}{2} \sum_{i=1}^{M} D \sigma^{i}(x) \sigma^{i}(x)+\sigma(x) \beta(t), & x(0)=x, \\
\dot{r}=l(x), & r(0)=r,
\end{array}
$$

and define the function $W^{\prime}(x, r):=W(x)+r$ with $r \in \mathbb{R}$. $\quad W^{\prime}$ is a viscosity supersolution to $\inf _{\beta \in \mathbb{R}^{M}} \max _{\alpha \in A}\left(-f(x, \alpha)+\frac{1}{2} \sum_{i=1}^{M} D \sigma^{i}(x) \sigma^{i}(x)-\sigma(x) \beta, l(x)\right) \cdot D W^{\prime}(x)=0$. If (27) holds for $W^{\prime}$ and for the trajectories of the augmented system, then it is immediate to show that it holds also for $W$ and the trajectories of the original system.

Moreover we may assume without loss of generality that $W$ is nonnegative and bounded. Indeed, consider a function $\rho: \mathbb{R} \rightarrow \mathbb{R}$ which is smooth, bounded, nonnegative and satisfies $0<\dot{\rho}<M$ for some $M$. The function $\rho(W)$ is still a viscosity supersolution to (26) with $l=0$ and is bounded and nonnegative. If we prove (27) for $\rho(W)$, then the same conclusion holds also for $W$. If it is not so, then there exists $x$ such that $W(x)<\inf _{\gamma \in \Gamma} \sup _{\beta \in \mathcal{B}} \sup _{t>0}[W(x(t ; \gamma(\beta), \beta))]$. So, it is possible to find $\varepsilon$ such that for every $\gamma \in \Gamma$ there exist $\beta \in \mathcal{B}$ and $t>0$ with $W(x)<W(x(t ; \gamma(\beta), \beta))+\varepsilon$. Now, applying $\rho$ to both sides and using the properties of this function, we get

$$
\rho(W(x))<\rho(W(x(t ; \gamma(\beta), \beta))+\varepsilon) \leq \rho(W(x(t ; \gamma(\beta), \beta)))+\bar{M} \varepsilon \quad \text { for some } \bar{M}>0,
$$

in contradiction with the assumption that $(27)$ holds for $\rho(W)$.

We start dividing the Hamiltonian by the factor $1+|b|$ to take care of unbounded disturbances: this leads to a reparametrization of the trajectories of the original dynamical system (DS). Indeed if $W$ is a supersolution to $(26)$ then it is also a supersolution to

$$
\inf _{\beta \in \mathbb{R}^{M}} \max _{\alpha \in A}[-F(x, \alpha, \beta) \cdot D W(x)]=0
$$

where

$$
F(x, \alpha, \beta):=\frac{f(x, \alpha)-\frac{1}{2} \sum_{i=1}^{M} D \sigma^{i}(x) \sigma^{i}(x)+\sigma(x) \beta}{1+|\beta|} .
$$

Observe that, by assumptions (4) and (5), $F$ satisfies the following uniform Lipschitz condition: there exists $L$ independent of $\alpha, \beta$ such that

$$
|F(x, \alpha, \beta)-F(y, \alpha, \beta)| \leq L|x-y|, \quad \text { for } x, y \in \mathbb{R}^{N}
$$

We consider now a sequence of continuous nonnegative functions $W_{n}$ such that $W=\sup _{n} W_{n}$. For every $\lambda \in(0,1)$ and every $n, W$ is a lower semicontinuous supersolution to the variational inequality of obstacle type (with obstacle $W_{n}$ )

$$
\min \left\{\lambda V(x)+\inf _{\beta \in \mathbb{R}^{M}} \max _{\alpha \in A}[-F(x, \alpha, \beta) \cdot D V(x)], V(x)-W_{n}(x)\right\}=0 .
$$


This equation satisfies a comparison principle among semicontinuous viscosity sub- and supersolutions (see [4, 17]). Then $W$ is greater than the value function of the differential game with optimal stopping criterion associated to the equation, that is,

$$
W(x) \geq \inf _{\gamma \in \Gamma} \sup _{\beta \in \mathcal{B}} \sup _{s \geq 0}\left[e^{-\lambda s} W_{n}(y(s ; \gamma(\beta), \beta))\right]
$$

where $y(\cdot ; \alpha, \beta)$ denotes the trajectory of the system:

$$
\left(D S^{\prime}\right)\left\{\begin{array}{l}
\dot{y}=F(y, \alpha, \beta) s>0 \\
y(0)=x
\end{array}\right.
$$

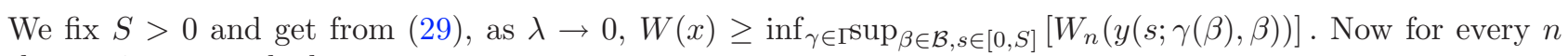
there exists $\gamma_{n}$ such that

$$
W(x)+1 / n \geq \sup _{\beta \in \mathcal{B}} \sup _{s \in[0, S]}\left[W_{n}\left(y\left(s ; \gamma_{n}(\beta), \beta\right)\right)\right] .
$$

Using the compactness of the product space $\left(L^{\infty}\left((0, S), A^{r}\right)\right)^{\mathcal{B}}$ and the convexity assumption (6), we get, passing to a subnet if necessary, that $\gamma_{n}$ converges to $\bar{\gamma}$ in the product topology of $\left(L^{\infty}\left((0, S), A^{r}\right)\right)^{\mathcal{B}}$ (for the detailed argument, we refer to [31] Lem. 2.2). It is easy to check, using the fact that $W$ is LSC and $W=\sup W_{n}$, that $\liminf \operatorname{in}_{n, z \rightarrow x} W_{n}(z)=W(x)$. From this and (30) we get, letting $n \rightarrow+\infty$,

$$
W(x) \geq \sup _{\beta \in \mathcal{B}} \sup _{s \in[0, S]}[W(y(s ; \bar{\gamma}(\beta), \beta))] \geq \inf _{\gamma \in \Gamma} \sup _{\beta \in \mathcal{B}} \sup _{s \in[0, S]}[W(y(s ; \gamma(\beta), \beta))] .
$$

We conclude by sending $S \rightarrow+\infty$ : this is done by constructing a global strategy repeating the previous argument on a sequence of intervals covering $[0,+\infty)$, we refer to $[30,31]$ for the details of this standard construction. Therefore we get

$$
W(x) \geq \inf _{\gamma \in \Gamma} \sup _{\beta \in \mathcal{B}} \sup _{s \geq 0}[W(y(s ; \gamma(\beta), \beta))] .
$$

For every $\beta \in \mathcal{B}$ we define a map $s_{\beta}(t)=\int_{0}^{t}(1+|\beta(u)|) \mathrm{d} u$ and denote with $t_{\beta}(s)$ the inverse of this map. Observe that the trajectories of the differential system $\left(\mathrm{DS}^{\prime}\right)$ coincide with the trajectories of the original system (DS) reparametrized using the new time variable $s$, so $y(s ; \gamma(\beta), \beta)$ coincides with $x\left(t_{\beta}(s) ; \gamma(\beta), \beta\right)$. Therefore we can rewrite (31) in terms of the trajectories of the original differential system (DS) and in terms of the time variable $t$ :

$$
W(x) \geq \inf _{\gamma \in \Gamma} \sup _{\beta \in \mathcal{B}} \sup _{t \geq 0}[W(x(t ; \gamma(\beta), \beta))] .
$$

This proves the assertion, since the other inequality is obtained by taking $t=0$.

\section{REFERENCES}

[1] S. Aida, S. Kusuoka and D. Stroock, On the support of Wiener functionals, Asymptotic problems in probability theory: Wiener functionals and asymptotics (Sanda/Kyoto, 1990), Pitman Res. Notes Math. Ser. 284, Longman Sci. Tech., Harlow (1993) $3-34$.

[2] J.-P. Aubin and G. Da Prato, The viability theorem for stochastic differential inclusions. Stochastic Anal. Appl. 16 (1998) $1-15$.

[3] J.-P.Aubin and H. Doss, Characterization of stochastic viability of any nonsmooth set involving its generalized contingent curvature. Stochastic Anal. Appl. 21 (2003) 955-981.

[4] M. Bardi and I. Capuzzo-Dolcetta, Optimal control and viscosity solutions of Hamilton-Jacobi-Bellman equations. Birkäuser, Boston (1997).

[5] M. Bardi and A. Cesaroni, Almost sure stabilizability of controlled degenerate diffusions. SIAM J. Control Optim. 44 (2005) $75-98$.

[6] M. Bardi and F. Da Lio, Propagation of maxima and strong maximum principle for viscosity solution of degenerate elliptic equations. I: Convex operators. Nonlinear Anal. 44 (2001) 991-1006. 
[7] M. Bardi and F. Da Lio, Propagation of maxima and strong maximum principle for fully nonlinear degenerate elliptic equations. II: Concave operators. Indiana Univ. Math. J. 52 (2003) 607-627.

[8] M. Bardi and P. Goatin, Invariant sets for controlled degenerate diffusions: a viscosity solutions approach, in Stochastic Analysis, Control, Optimization and Applications: A Volume in Honor of W.H. Fleming, W.M. McEneaney, G.G. Yin and Q. Zhang Eds., Birkhäuser, Boston (1999) 191-208.

[9] M. Bardi and R. Jensen, A geometric characterization of viable sets for controlled degenerate diffusions. Set-Valued Anal. 10 (2002) 129-141.

[10] T. Başar and P. Bernhard, $H^{\infty}$-optimal control and related minimax design problems. A dynamic game approach, 2nd edn., Birkhäuser, Boston (1995).

[11] G. Ben Arous, M. Grădinaru and M. Ledoux, Hölder norms and the support theorem for diffusions. Ann. Inst. H. Poincaré Probab. Statist. 30 (1994) 415-436.

[12] P. Bernhard, Robust control approach to option pricing, including transaction costs, in Advances in dynamic games, Ann. Internat. Soc. Dynam. Games 7, Birkhäuser, Boston (2005) 391-416.

[13] R. Buckdahn, S. Peng, M. Quincampoix and C. Rainer, Existence of stochastic control under state constraints. C. R. Acad. Sci. Paris Sér. I Math. 327 (1998) 17-22.

[14] P. Cardaliaguet, A differential game with two players and one target. SIAM J. Control Optim. 34 (1996) 1441-1460.

[15] A. Cesaroni, Stability properties of controlled diffusion processes via viscosity methods. Ph.D. thesis, University of Padova (2004).

[16] A. Cesaroni, A converse Lyapunov theorem for almost sure stabilizability. Systems Control Lett. 55 (2006) $992-998$.

[17] M.C. Crandall, H. Ishii and P.L. Lions, User's guide to viscosity solutions of second order partial differential equations. Bull. Amer. Math. Soc. 27 (1992) 1-67.

[18] G. Da Prato and H. Frankowska, Invariance of stochastic control systems with deterministic arguments. J. Diff. Equ. 200 (2004) 18-52.

[19] H. Doss, Liens entre équations différentielles stochastiques et ordinaires. Ann. Inst. H. Poincaré Sect. B (N.S.) 13 (1977) $99-125$.

[20] W.H. Fleming and H.M. Soner, Controlled Markov Process and Viscosity Solutions. Springer-Verlag, New York (1993).

[21] R.A. Freeman and P.V. Kokotovic: Robust nonlinear control design. State-space and Lyapunov techniques. Birkäuser, Boston (1996).

[22] U.G. Haussmann and J.P. Lepeltier, On the existence of optimal controls. SIAM J. Control Optim. 28 (1990) 851-902.

[23] Y. Lin, E.D. Sontag, Control-Lyapunov universal formulas for restricted inputs. Control Theory Adv. Tech. 10 (1995) 19812004 .

[24] A. Millet and M. Sanz-Solé, A simple proof of the support theorem for diffusion processes, Séminaire de Probabilités, XXVIII, Lect. Notes Math. 1583, Springer, Berlin (1994) 36-48.

[25] G.J. Olsder, Differential game-theoretic thoughts on option pricing and transaction costs. Int. Game Theory Rev. 2 (2000) $209-228$.

[26] H.M. Soner and N. Touzi, Dynamic programming for stochastic target problems and geometric flow. J. Eur. Math. Soc. 4 (2002) 201-236.

[27] H.M. Soner and N. Touzi, A stochastic representation for the level set equations. Comm. Part. Diff. Equ. 27 (2002) $2031-2053$.

[28] P. Soravia, Pursuit-evasion problems and viscosity solutions of Isaacs equations. SIAM J. Control. Optim. 31 (1993) 604-623.

[29] P. Soravia, Stability of dynamical systems with competitive controls: the degenerate case. J. Math. Anal. Appl. 191 (1995) 428-449.

[30] P. Soravia, $\mathcal{H}_{\infty}$ control of nonlinear systems: differential games and viscosity solutions. SIAM J. Control Optim. 34 (1996) 1071-1097.

[31] P. Soravia, Equivalence between nonlinear $\mathcal{H}_{\infty}$ control problems and existence of viscosity solutions of Hamilton-Jacobi-Isaacs equations. Appl. Math. Optim. 39 (1999) 17-32.

[32] D.W. Stroock and S.R.S. Varadhan, On the support of diffusion processes with applications to the strong maximum principle, in Proceedings of the Sixth Berkeley Symposium on Mathematical Statistics and Probability (Univ. California, Berkeley, Calif., 1970/1971), Vol. III: Probability theory, Univ. California Press, Berkeley (1972) 333-359.

[33] D.W. Stroock and S.R.S. Varadhan, On degenerate elliptic-parabolic operators of second order and their associated diffusions. Comm. Pure Appl. Math. 25 (1972) 651-713.

[34] H.J. Sussmann, On the gap between deterministic and stochastic ordinary differential equations. Ann. Probability 6 (1978) 19-41. 